\title{
Insulitic profiles in T1DM
}

Insulitis, the infiltration of lymphocytes and consequent inflammation of pancreatic islets, was thought to occur in a fairly uniform manner in type 1 diabetes mellitus (T1DM). However, a newly-identified feature in the lymphocyte-specific profile of insulitis might change our understanding of the disease.

The investigators, reporting in Diabetes, found that patients with new-onset T1DM had variable numbers of $\mathrm{CD} 20^{+} \mathrm{B}$ lymphocytes in pancreatic islets, called $\mathrm{CD} 2 \mathrm{OHi}$ and CD20Lo, which corresponded to the age of disease onset. Those individuals diagnosed with T1DM before the age of 7 years had a ratio of $\mathrm{CD}_{20}{ }^{+}$to $\mathrm{CD}_{4}{ }^{+}$cells greater than one $(\mathrm{CD} 2 \mathrm{OHi})$; those diagnosed after the age of 13 years had a ratio of $\mathrm{CD} 20^{+}$to $\mathrm{CD}^{+}$less than one
(CD20Lo). Cytotoxic CD8 ${ }^{+}$cells were present, regardless of the number of CD20+ cells.

"These two phenotypes are not distributed randomly among patients with type 1 diabetes but segregate with age at onset," explains Noel Morgan who helped lead the study. Interestingly, this association was also identified in samples from the nPOD study from the USA and DiViD in Norway, suggesting that this profile of insulitis is indicative of a general T1DM mechanism.

The different lymphocyte profile might also have a functional consequence on the aggressiveness of T1DM. Patients diagnosed at a younger age had fewer residual insulin-containing islets than patients in older age groups $(14 \pm 3 \%$ versus
$39 \pm 4 \%$ for patients diagnosed aged 1 to 6 and $>13$ years respectively; $P<0.001)$.

The team hope that stratifying patients based on their insulitic profile will aid the design of new therapies for T1DM. "We are interested in understanding whether $\mathrm{CD} 20^{+}$cells actively promote the cytotoxic actions of $\mathrm{CD} 8^{+} \mathrm{T}$ cells," adds Morgan. "This understanding might then provide a means to intervene in the process in order to reduce the rate of $\beta$-cell loss in relevant patients."

Tim Geach

ORIGINAL ARTICLE Leete, P. et al. Differentia insulitic profiles determine the extent of beta cell destruction and the age at onset of type 1 diabetes. Diabetes http://dx.doi.org/10.2337/ $\underline{\mathrm{db} 15-1615}$ 